\title{
Impact of Dietary and Other Factors Including lodine Sufficiency Status on the Levels of Urinary Phytoestrogens
}

Keywords: Daidzein; Genistein; Enterodiol; Enterolactone; Smoking lodine

\author{
Abstract \\ Background and aims: Data from National Health and Nutrition \\ Examination Survey for 2007-2010 were used to evaluate factors that \\ affect observed variability in urinary phytoestrogens.
}

Methods: Data for six phytoestrogen variables, namely, daidzein (DAZ), o-desmethylangolensin (DMA), equol (EQU), enterodio (ETD), enterolactone (ETL), and genistein (GNS) were available for analyses. Regression models for log 10 transformed values of these six phytoestrogens as dependent variables and gender, race/ethnicity, smoking status, iodine sufficiency status, body mass index, poverty income ratio, urine creatinine, consumption of selected foods, and selected total nutrient variables as independent variables were fitted.

Results: Males had statistically significantly higher levels of DAZ, DMA, EQU, ETD, and ETL than females ( $\mathrm{p} \leq 0.03$ ). Non-Hispanic whites had statistically significantly higher levels of DMA, EQU, and ETD ( $p<$ 0.01 ) than Mexican Americans. NHW had statistically significantly higher levels of EQU and ETD ( $p<0.01)$ than non-Hispanic blacks. Smoking was associated with $42.9 \%, 18.2 \%, 41 \%$, and $72 \%$ lower levels of DMA EQU, ETD, and ETL respectively as compared to nonsmokers. Iodine deficiency was associated with $47 \%, 55.6 \%, 88.6 \%, 4.5 \%, 17.8 \%$, and $29.2 \%$ lower levels of DAZ, DMA, EQU, ETD, ETL, and GNS respectively. Levels of EQU were positively associated with consumptions of milk products and seeds. Consumption of legumes, nuts, and grains had positive association with DAZ, DMA, and GNS. Levels of DAZ, DMA and ETL were positively associated with consumption of eggs. Finally, there were positive associations between the consumptions of fish and seeds with the levels of ETD.

Conclusion: In conclusion, both smoking and iodine deficiency may affect metabolism of selected phytoestrogens.

\section{Introduction}

Phytoestrogens are plant-derived naturally occurring phenolic compounds which have estrogenic effects [1]. Isoflavones, lignans, and coumestrans are three major classes of phytoestrogens [2,3]. Isoflavones include genistein (GNS) and daidzein (DAZ), and they are found in the highest amounts in soybeans, miso, tofu, and legumes [2,4]. Lignans are constituents of linseed and sesame seeds and in lower concentrations; in seed oils, whole grain cereals, beans, and other fruits and vegetables $[4,5]$.

Decreased incidence of diabetes, heart disease, and breast cancer has been reported to be associated with the consumption of phytoestrogens. For example, as compared to non-consumers, consumption of phytoestrogens has been shown to be associated with decreased incidence of diabetes and heart disease by Zhang et al. and Villegas et al. [6,7]. Similarly as compared to non-consumers of phytoestrogens, consumption of phytoestrogens was found to

\section{Journal of}

Nutrition and Health

\author{
Ram B. Jain* \\ Private Consultant, Clinical Data Consultants, Dacula, Georgia, \\ USA \\ ${ }^{*}$ Address for Correspondence \\ Ram B. Jain, Private Consultant, Clinical Data Consultants, Dacula, Ga \\ 30019, USA, Tel: 001-910-729-1049; E-mail: Jain.ram.b@gmail.com \\ Submission: 16 February, 2016 \\ Accepted: 11 April, 2016 \\ Published: 16 April, 2016 \\ Copyright: () 2016 Jain RB. This is an open access article distributed \\ under the Creative Commons Attribution License, which permits \\ unrestricted use, distribution, and reproduction in any medium, provided \\ the original work is properly cited. \\ Reviewed \& Approved by: Dr. Charalampos Proestos, \\ Department of Chemistry, National and Kapodistrian University of \\ Athens, Greece
}

be associated with reduced risk of breast cancer incidence in Asian populations [8,9]. In a meta-analysis, relative risk of ovarian cancer among those who consumed isoflavones as compared to those who did not consume isoflavones was reported to be 0.63 and relative risk among those who consumed soy foods as compared to those who did not consume soy foods was estimated to be 0.51 [10]. When compared with non-consumers, relative risks of prostate cancer associated with the consumptions of soy and isoflavones were shown to be 0.74 and 0.88 respectively [11].

Jeng et al. used cross-sectional data from National Health and Nutrition Examination Survey (NHANES) for the years 2009-2010 for children and adolescents aged 6-18 years to evaluate the associations between the levels of phytoestrogens and metabolic disturbances [12]. These authors found that the levels of enterolactone (ETL) and equol (EQU) were inversely related to systolic blood pressure and the levels of daidzein (DAZ), EQU, genistein (GNS), o-desmethylangolensin (DMA) were inversely related to the levels of triglyceride [12]. On the other hand, Jeng et al. also found an inverse relationship between the levels of high density lipoproteins and GNS and DMA. Thus, certain phytoestrogens were found to contribute both negatively and positively to metabolic processes [12]. In postmenopausal females with metabolic syndrome, soy diet was associated with decreased diastolic blood pressure, triglyceride levels, and C-reactive protein [13]. However, among those without metabolic syndrome, only EQU producers had reductions in diastolic blood pressure and C-reactive protein [13]. Thus, in selected populations, diets rich in phytoestrogens make positive contributions to lipid metabolism.

Ryback et al. used data from NHANES for the years 2003-2006 for adults aged $\geq 20$ years to evaluate associations of sociodemographic and life style factors with the observed levels of DAZ, EQU, ETL, ETD, GNS and DMA in urine [14]. Smokers were found to have lower levels of ETL and DMA by at least 25\% when compared with nonsmokers [14]. As compared to non-consumers of alcohol, consumers of 1 daily drink of alcohol were found to have lower levels of EQU and DMA by about $18-21 \%$ [14]. However, these authors did not consider the role 
Citation: Jain RB. Impact of Dietary and Other Factors Including lodine Sufficiency Status on the Levels of Urinary Phytoestrogens. J Nutri Health. 2016;2(1): 7

that iodine deficiency may play in observed levels of phytoestrogens.

Since, to the best of my knowledge, association between iodine and phytoestrogen levels has not been evaluated in a nationally representative sample of adults, this study was undertaken to evaluate the association between iodine sufficiency status as defined by the observed urinary iodine levels and metabolism of six phytoestrogens as defined by their observed levels in urine. Data from NHANES for the years 2007-2010 were selected for this purpose.

\section{Materials and Methods}

NHANES (www.cdc.gov/nchs/nhanes.htm) data for those aged $\geq$ 20 years for the years 2007-2010 from demographic, phytoestrogen, serum cotinine, urinary iodine, first day individual food files, and first day total nutrient food files were downloaded and match merged. NHANES uses a complex, stratified, multistage, probability sampling designed as representative of the civilian, non-institutionalized U.S. population based on age, gender, and race/ethnicity. Sampling weights are created in NHANES to account for the complex survey design, including oversampling, survey non-response, and poststratification.

Data were available for 6 urinary phytoestrogens, namely, daidzein (DAZ), o-desmethylangolensin (DMA), equol (EQU), enterodiol (ETD), enterolactone (ETL), and genistein (GNS). Percent values at or above the limit of detection (LOD) for DAZ, DMA, EQU, ETD, ETL, and GNS were 99.9\%, 93.7\%, 99.7\%, 99.3\%, 99.9\%, and $100 \%$ respectively. LODs for DAZ, DMA, EQU, ETD, ETL, and GNS were $0.40,0.20,0.06,0.04,0.01$, and $0.20 \mathrm{ng} / \mathrm{mL}$ respectively All values $<$ LOD were imputed as LOD/Sqrt (2).

Urine samples were collected by the Centers for Disease Control and Prevention and were assayed at the Division of Laboratory Sciences of the National Center for Environmental Health of the Centers for Disease Control and Prevention. Briefly, high performance liquid chromatography-atmospheric pressure photoionization-tandem mass spectrometry (HPLC-APPI-MS/MS) was sued for the quantitative detection of genistein, daidzein, equol, O-desmethylangolensin, enterodiol, and enterolactone. Detailed laboratory methods to measure phytoestrogens are available at http:// wwwn.cdc.gov/nchs/nhanes/2009-2010/PHYTO_F.htm.

Using data from the individual food files, data from nine major food groups specified by United States Department of Agriculture $[15,16]$ in their food and nutrition database were used to create 17 food groups for the purpose of this study. The conversion of 9 original USDA food groups to 17 new groups was accomplished by classifying these groups based on USDA food codes. USDA food code is an 8-digit code. Only first two (USDA-2), first three (USDA-3), and four digits (USDA-4) were used to re-classify the food groups used in this study. Table 1 provides details on how 17 food groups used in this study were created and how they are related to original USDA food groups. The only data used for each of the 17 food groups for this study was the amount of each food consumed by the participants. All those who were not reported to have consumed food in a particular food group were assumed to have consumed zero gram of food in that food group. From the total nutrient intake data files, total amount of alcohol, caffeine, cholesterol, fiber, folate, sugar, and fat consumed in grams, and total calories consumed in kilo-calories were used.

The total number of participants aged $\geq 20$ years available for analysis, after removing observations with missing data for dependent and independent variables (as defined later on), was 2962. Detailed sample sizes were: $\mathrm{N}_{\text {Males }}=1462, \mathrm{~N}_{\text {Females }}=1500, \mathrm{~N}_{\text {Non-Hispanic White }}=1457$, $\mathrm{N}_{\text {Non-Hispanic Black }}=548, \quad \mathrm{~N}_{\text {Mexican American }}=561, \quad \mathrm{~N}_{\text {Other race/thnicities }}=396$, $\mathrm{N}_{\text {Nonsmoker }}=2207, \mathrm{~N}_{\text {Smoker }}=755, \mathrm{~N}_{\text {Iodine Deficient }}=950$, and $\mathrm{N}_{\text {Iodine Replete }}=2012$. Those with urinary iodine concentration $<100 \mu \mathrm{g} / \mathrm{L}$ were defined as iodine deficient and those with urinary iodine concentration $>=$ $100 \mu \mathrm{g} / \mathrm{L}$ were defined as iodine replete. Those with serum cotinine levels $<10 \mathrm{ng} / \mathrm{mL}$ were defined as nonsmokers and those with serum cotinine levels $>=10 \mathrm{ng} / \mathrm{mL}$ were defined as smokers.

The author analyzed all raw downloaded data from NHANES by using SAS v. 9.3 (www.sas.com, SAS Institute for Advanced Analytics, Cary, North Carolina, USA). In order to assess factors affecting observed phytoestrogen levels, using SAS Proc SURVEYREG, multivariate regression models were fitted with $\log 10$ transformed values of urinary phytoestrogen levels as dependent variables. A total of four regression models (Model 1 through Model 4) were fitted in a sequential fashion for each of the six phytoestrogens. Thus, a total of 24 regression models were fitted. The independent variables included in Model 1 were: gender (males, females), race/ ethnicity (non-Hispanic white or NHW, non-Hispanic black or NHB, Mexican American or MA, other unclassified race/ethnicities or OTH), as categorical variables; and age, body mass index (BMI), family poverty income ratio (PIR), NHANES survey year, namely, 2007-2008 and 2009-2010 labeled as numeric 1 and 2 respectively, and urine creatinine as continuous variables. Model 2 included all the independent variables in Model 1 and smoking status (nonsmoker, smoker) and iodine sufficiency status (iodine deficient, iodine replete) as categorical variables. Model 3 included all the independent variables in Model 2 and, in addition, each of the 17 daily food consumption variables as listed in Table 1 were added as continuous variables but only those with $p \leq 0.10$ were retained in the model. Finally, Model 4 included all the independent variables already in Model 3 and in addition, total nutrient variables, namely, total amount of alcohol, caffeine, cholesterol, fiber, folate, sugar, and fat consumed in grams, and total calories consumed in kilo-calories were added in the model but only those with $p \leq 0.10$ were retained in the final model. All results generated by regression models and as presented in this communication are based on final fitted Model 4.

It should be noted that all analyses incorporated variables to reflect adjustments that were necessary to account for complex survey design, namely, stratification and clustering. Information on stratification and clustering within each stratum is provided in all publically released NHANES datasets as variables named SDMVSTRA and SDMVPSU respectively. SAS statements STRATA and CLUSTER allow specification of these design variables.

\section{Results}

\section{Relative contributions to regression model $\mathbf{R}^{2}$}

With age, gender, race/ethnicity, BMI, PIR, urine creatinine, and survey year only in the model, $\mathrm{R}^{2}$ varied from $4.51 \%$ for the model for DMA to $13.64 \%$ for the model for EQU (Table 2). The addition of smoking and iodine sufficiency statuses to the models raised $\mathrm{R}^{2}$ from 
Citation: Jain RB. Impact of Dietary and Other Factors Including lodine Sufficiency Status on the Levels of Urinary Phytoestrogens. J Nutri Health. 2016;2(1): 7.

\section{ISSN: 2469-4185}

Table 1: Conversion algorithm used to convert nine original United States Department of Agriculture (USDA) food groups to 17 new food groups.

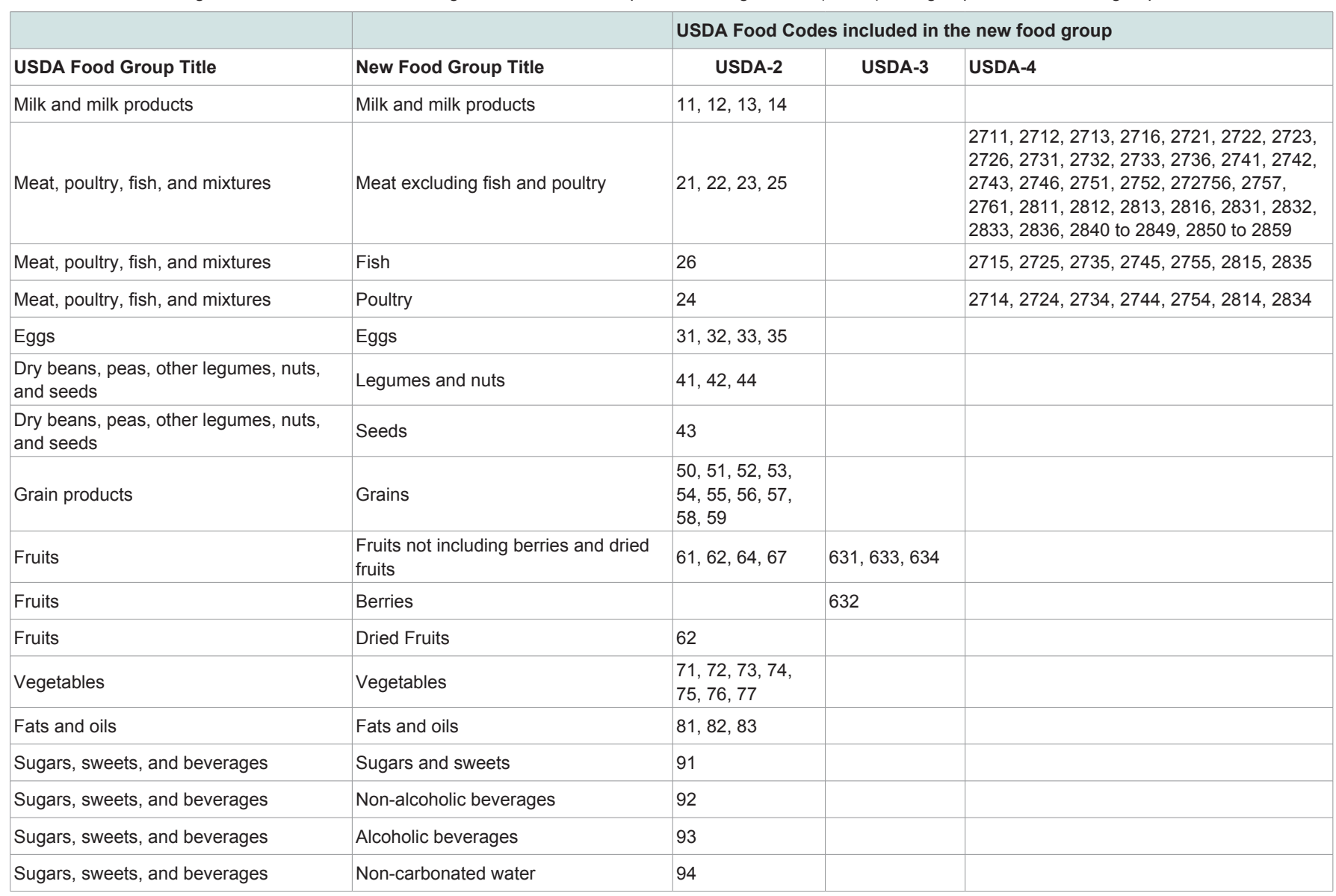

Table 2: Relative contribution of various independent variables to the model $\mathrm{R}^{2}$. Based on analysis of data from National Health and Nutrition Examination Survey 2007-2010.

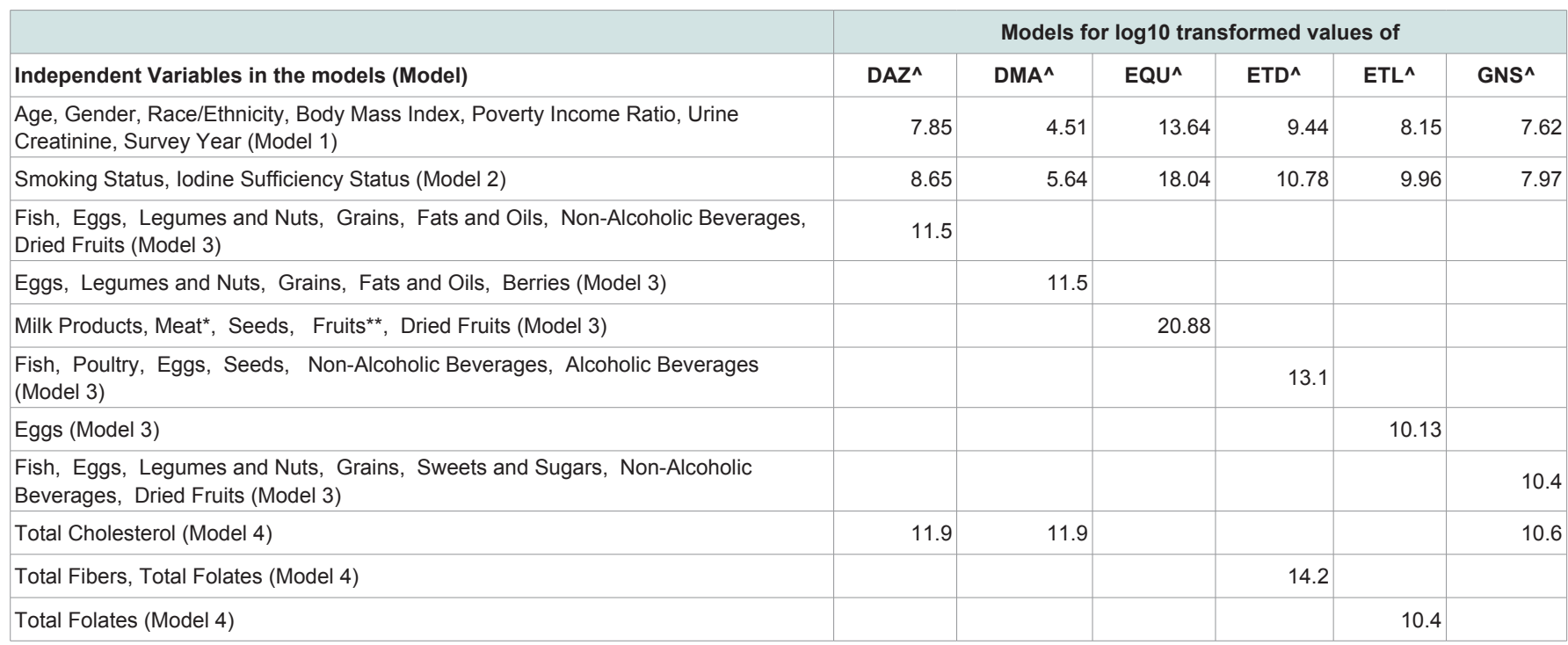

*not including fish and poultry

${ }^{* *}$ not including berries and dried fruits

${ }^{\wedge} \mathrm{DAZ}=$ daidzein, $\mathrm{DMA}=\mathrm{o}$-desmethylangolensin, $E Q U=$ equol, $E T D=$ enterodiol, $E T L=$ enterolactone, $G N S=$ genistein

$0.35 \%$ for GNS to $4.4 \%$ for EQU. With age, gender, race/ethnicity, BMI, PIR, urine creatinine, survey year, smoking and iodine sufficiency statuses in the models, smoking and iodine sufficiency statuses contributed $9.2 \%, 20 \%, 24.4 \%, 12.4 \%, 18.2 \%$, and $4.4 \%$ 
Citation: Jain RB. Impact of Dietary and Other Factors Including lodine Sufficiency Status on the Levels of Urinary Phytoestrogens. J Nutri Health. 2016;2(1): 7 .

ISSN: $2469-4185$

Table 3: Regression coefficients with associated p-values for continuous variable used as independent variables. Based on analysis of data from National Health and Nutrition Examination Survey 2007-2010.

\begin{tabular}{|c|c|c|c|c|c|c|}
\hline \multirow[b]{2}{*}{ Independent Variable } & \multicolumn{6}{|c|}{ Dependent Variable was Log10 of } \\
\hline & Daidzein & $\begin{array}{l}\text { O-Desmethyl- } \\
\text { angolensin }\end{array}$ & Equol & Enterodiol & Enteriolactone & Genistein \\
\hline Age & $0.0012(0.27)$ & $0.0002(0.91)$ & $-0.0004(0.67)$ & $0.0028(<0.01)$ & $0.006(<0.01)$ & $0.0024(0.047)$ \\
\hline Survey Year & $0.0034(0.93)$ & $0.0056(0.91)$ & $0.0362(0.44)$ & $0.0638(0.08)$ & $-0.0098(0.82)$ & $-0.0066(0.87)$ \\
\hline Body Mass Index & $-0.0008(0.79)$ & $-0.0034(0.34)$ & $-0.0033(0.09)$ & $-0.0079(0.01)$ & $-0.0163(<0.01)$ & $-0.003(0.29)$ \\
\hline Poverty Income Ratio & $0.0111(0.34)$ & $0.0307(0.11)$ & $0.0181(0.06)$ & $0.0385(<0.01)$ & $0.0427(<0.01)$ & $0.0009(0.93)$ \\
\hline Urine Creatinine & $0.0027(<0.01)$ & $0.0023(<0.01)$ & $0.0023(<0.01)$ & $0.0031(<0.01)$ & $0.0022(<0.01)$ & $0.0027(<0.01)$ \\
\hline Milk and Milk Products & $\mathrm{NIM}^{*}$ & $\mathrm{NIM}^{*}$ & $0.0004(<0.01)$ & $\mathrm{NIM}^{*}$ & $\mathrm{NIM}^{*}$ & $\mathrm{NIM}^{*}$ \\
\hline Meat excluding Fish and Poultry & $\mathrm{NIM}^{*}$ & $\mathrm{NIM}^{*}$ & $-0.0001(0.14)$ & $\mathrm{NIM}^{*}$ & $\mathrm{NIM}^{*}$ & $\mathrm{NIM}^{*}$ \\
\hline Fish & $0.0003(0.12)$ & $\mathrm{NIM}^{*}$ & $\mathrm{NIM}^{*}$ & $0.0003(0.03)$ & $\mathrm{NIM}^{*}$ & $0.0004(0.08)$ \\
\hline Poultry & $\mathrm{NIM}^{*}$ & $\mathrm{NIM}^{*}$ & $\mathrm{NIM}^{*}$ & $0.0002(0.17)$ & $\mathrm{NIM}^{*}$ & $\mathrm{NIM}^{*}$ \\
\hline Legumes and Nuts & $0.0009(<0.01)$ & $0.0012(<0.01)$ & $\mathrm{NIM}^{*}$ & $\mathrm{NIM}^{*}$ & $\mathrm{NIM}^{*}$ & $0.0006(<0.01)$ \\
\hline Seeds & $\mathrm{NIM}^{*}$ & $\mathrm{NIM}^{*}$ & $0.0041(0.03)$ & $0.0071(<0.01)$ & $\mathrm{NIM}^{*}$ & $\mathrm{NIM}^{*}$ \\
\hline Grains & $0.0004(<0.01)$ & $0.0003(<0.01)$ & $\mathrm{NIM}^{*}$ & $\mathrm{NIM}^{*}$ & $\mathrm{NIM}^{*}$ & $0.0004(<0.01)$ \\
\hline Fruits excluding Berries and Dried Fruits & $\mathrm{NIM}^{*}$ & $\mathrm{NIM}^{*}$ & $-0.0002(0.01)$ & $\mathrm{NIM}^{*}$ & $\mathrm{NIM}^{*}$ & $\mathrm{NIM}^{*}$ \\
\hline Vegetables & $\mathrm{NIM}^{*}$ & $\mathrm{NIM}^{*}$ & $\mathrm{NIM}^{*}$ & $\mathrm{NIM}^{*}$ & $\mathrm{NIM}^{*}$ & $\mathrm{NIM}^{*}$ \\
\hline Fats and Oils & $0.0022(0.02)$ & $0.0026(<0.01)$ & $\mathrm{NIM}^{*}$ & $\mathrm{NIM}^{*}$ & $\mathrm{NIM}^{*}$ & $\mathrm{NIM}^{*}$ \\
\hline Sweets and Sugars & $\mathrm{NIM}^{*}$ & $\mathrm{NIM}^{*}$ & $\mathrm{NIM}^{*}$ & $\mathrm{NIM}^{*}$ & $\mathrm{NIM}^{*}$ & $-0.0004(0.08)$ \\
\hline Non-Alcoholic Beverages & $-0.00002(0.38)$ & $\mathrm{NIM}^{*}$ & $\mathrm{NIM}^{*}$ & $-0.0001(<0.01)$ & $\mathrm{NIM}^{*}$ & $-0.00004(0.051)$ \\
\hline Alcoholic Beverages & $\mathrm{NIM}^{*}$ & $\mathrm{NIM}^{*}$ & $\mathrm{NIM}^{*}$ & $0.0001(<0.01)$ & $\mathrm{NIM}^{*}$ & $\mathrm{NIM}^{*}$ \\
\hline Berries & $\mathrm{NIM}^{*}$ & $0.0011(0.1)$ & $\mathrm{NIM}^{*}$ & $\mathrm{NIM}^{*}$ & $\mathrm{NIM}^{*}$ & $\mathrm{NIM}^{*}$ \\
\hline Dried Fruits & $0.0046(0.1)$ & $\mathrm{NIM}^{*}$ & $0.004(0.09)$ & $\mathrm{NIM}^{*}$ & $\mathrm{NIM}^{*}$ & $0.0048(0.08)$ \\
\hline Total Fiber & $\mathrm{NIM}^{*}$ & $\mathrm{NIM}^{*}$ & $\mathrm{NIM}^{*}$ & $0.0075(<0.01)$ & $\mathrm{NIM}^{*}$ & $\mathrm{NIM}^{*}$ \\
\hline Total Folate & $\mathrm{NIM}^{*}$ & $\mathrm{NIM}^{*}$ & $\mathrm{NIM}^{*}$ & $0.0001(0.23)$ & $0.0002(<0.01)$ & $\mathrm{NIM}^{*}$ \\
\hline
\end{tabular}

${ }^{*} \mathrm{NIM}=$ Not in Model

(Table 2) to the explained variance. Addition of the consumption of individual food variables added $\mathrm{R}^{2}$ from $0.17 \%$ for the model for ETL to $5.86 \%$ for the model for DMA. With individual food consumption variables also in the model, individual food consumption variables themselves accounted for $51 \%$ of the explained variance for DMA. Increase in $\mathrm{R}^{2}$ due to the addition of total nutrient variables was $0 \%$ for the model for EQU to $1.1 \%$ for the model for ETD.

\section{Association between phytoestrogens and continuous independent variables in the models}

Levels of ETD $(\beta=0.0028, \mathrm{p}<0.01)$, ETL $(\beta=0.006, \mathrm{p}<0.01)$, and GNS ( $\beta=0.0024, p=0.047)$ increased with crease in age (Table 3$)$. Levels of ETD $(\beta=-0.0079, p=0.01)$ and ETL $(\beta=-0.0163, p<0.01)$ decreased with increase in BMI. There was a positive association between PIR and ETD $(\beta=0.0385, p<0.01)$ and ETL $(\beta=0.0427$, $p<0.01)$. Increased consumption of legumes and nuts $(\beta=0.0009$, $p<0.01)$, eggs $(\beta=0.001, p<0.01)$, grains $(\beta=0.0004, p<0.01)$, fats and oils $(\beta=0.0022, p=0.02)$ was associated with increased levels of DAZ. Increased consumption of legumes and nuts $(\beta=0.0012$, $\mathrm{p}<0.01)$, eggs $(\beta=0.0015, \mathrm{p}<0.01)$, grains $(\beta=0.0003, \mathrm{p}<0.01)$, fats and oils $(\beta=0.0026, p<0.01)$ was associated with increased levels of DMA. There was a positive association between the levels of EQU and consumption of milk and milk products $(\beta=0.0004, \mathrm{p}<0.01)$ and seeds $(\beta=0.0041, p=0.03)$ but increased consumption of fruits excluding berries and dried fruits was associated with decreased levels of EQU $(\beta=-0.0002, p=0.01)$. Increase in the consumption of fish ( $\beta=$ $0.0003, p=0.03)$, seeds $(\beta=0.0071, p<0.01)$, and alcoholic beverages $(\beta=0.0001, p<0.01)$ was associated with increased levels of ETD. In addition, increased consumption of non-alcoholic beverages $(\beta=$ $0.0001, \mathrm{p}<0.01$ ) was associated with decreased levels of ETD. There was a positive association between the consumption of eggs and ETL $(\beta=0.0005, p=0.03)$. Consumption of both legumes and nuts $(\beta=$ $0.0006, p<0.01)$ and grains $(\beta=0.0004, p<0.01)$ was associated with increase in the levels of GNS.

Increased levels of DAZ $(\beta=-0.0003, \mathrm{p}<0.01)$, DMA $(\beta=-$ $0.0004, p<0.01)$, and GNS $(\beta=-0.0002, p=0.02)$ were associated with decreased levels of total cholesterol. There was a positive association 
Citation: Jain RB. Impact of Dietary and Other Factors Including lodine Sufficiency Status on the Levels of Urinary Phytoestrogens. J Nutri Health. 2016;2(1): 7

ISSN: $2469-4185$

Table 4: Adjusted geometric means with $95 \%$ confidence intervals (given in parenthesis) in $\mathrm{ng} / \mathrm{mL}$ for selected phytoestrogens by gender, race/ethnicity, smoking and iodine sufficiency status. Based on analysis of data from National Health and Nutrition Examination Survey 2007-2010.

\begin{tabular}{|c|c|c|c|c|c|c|}
\hline & Daidzein* $^{*}$ & $\underline{\text { O-Desmethylangolensin }}{ }^{* *}$ & Equol ${ }^{* * *}$ & Enterodiol^ & Enteriolactone ${ }^{\wedge \wedge}$ & 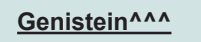 \\
\hline Males $(M)^{\&}$ & $66(54-80.6)$ & $4.2(3.3-5.2)$ & $5.2(4.3-6.2)$ & $37.4(30.6-45.8)$ & $192.3(161.8-228.5)$ & $32.8(26.9-40)$ \\
\hline Females $(F)^{\&}$ & $52.9(44.6-62.7)$ & $2.7(2.2-3.2)$ & $4.5(3.8-5.2)$ & $23.4(19.3-28.3)$ & $155.8(128.5-189)$ & $28.1(23.4-33.7)$ \\
\hline Non-Hispanic Whites (NHW)\# & $59.5(49.6-71.2)$ & $3.8(3.2-4.5)$ & $6.5(5.6-7.7)$ & $38.3(32.8-44.7)$ & $201.7(174.8-232.7)$ & $30.5(25.1-37)$ \\
\hline Non-Hispanic Blacks (NHB)\# & $58.3(45.3-75.1)$ & $4.1(3.1-5.5)$ & $4.3(3.5-5.3)$ & $24.6(19.9-30.3)$ & $166.2(124.9-221.1)$ & $26.8(21.7-33)$ \\
\hline Mexican Americans (MA) ${ }^{\#}$ & $47.1(36.4-61.1)$ & $2.3(1.7-3)$ & $3.8(3.1-4.7)$ & $26.5(21.5-32.7)$ & $217.7(182.1-260.4)$ & $25.5(19.8-32.7)$ \\
\hline Others $(\mathrm{OTH})^{\#}$ & $74.4(55.4-100)$ & $3.6(2.5-5)$ & $5(3.7-6.7)$ & $30.6(22.7-41.2)$ & $123(86.4-175.1)$ & $40.9(30.2-55.3)$ \\
\hline Nonsmoker (NSM)\&\& & $59.1(49.5-70.5)$ & $4(3.4-4.8)$ & $5.2(4.5-6)$ & $35.1(29.7-41.6)$ & $227.1(193.5-266.4)$ & $29.2(24.8-34.3)$ \\
\hline Smoker (SM) \&\& & $59(47.5-73.3)$ & $2.8(2.2-3.4)$ & $4.4(3.6-5.4)$ & $24.9(20.5-30.3)$ & $132(107-162.7)$ & $31.6(25.2-39.6)$ \\
\hline lodine Deficient (IOD) & $48.7(40.3-59)$ & $2.7(2.2-3.2)$ & $3.5(2.9-4.2)$ & $28.9(23.6-35.4)$ & $159.5(129.3-196.7)$ & $26.7(21.8-32.8)$ \\
\hline lodine Replete (IOR) \#\# & $71.6(59.2-86.4)$ & $4.2(3.4-5.1)$ & $6.6(5.7-7.8)$ & $30.2(25.3-36.1)$ & $187.9(159.2-221.7)$ & $34.5(28.8-41.3)$ \\
\hline
\end{tabular}

${ }^{*} M>F(p<0.01), M A<O T H(p<0.01), I O D<I O R(p<0.01)$

${ }^{* *} \mathrm{M}>\mathrm{F}(p<0.01), \mathrm{NHW}>\mathrm{MA}(\mathrm{p}<0.01), \mathrm{NHB}>\mathrm{MA}(\mathrm{p}<0.01), \mathrm{MA}<\mathrm{OTH}(\mathrm{p}=0.02), \mathrm{NSM}>\mathrm{SM}(\mathrm{p}=0.01), \mathrm{IOD}<\mathrm{IOR}(p=0.01)$

***M $>F(p=0.02), N H W>N H B(p<0.01), N H W>M A(p<0.01), N S M>S M(p=0.04), I O D<I O R(p<0.01)$

${ }^{\wedge} M>F(p<0.01), N H W>N H B(p<0.01), N H W>M A(p<0.01), N S M>S M(p<0.01)$

${ }^{\wedge \wedge} \mathrm{M}>\mathrm{F}(\mathrm{p}=0.03), \mathrm{NHW}>\mathrm{OTH}(\mathrm{p}<0.01), \mathrm{MA}>\mathrm{OTH}(\mathrm{p}<0.01), \mathrm{NSM}>\mathrm{SM}(\mathrm{p}<0.01)$

$\wedge \wedge \wedge \mathrm{NHB}<\mathrm{OTH}(\mathrm{p}=0.01), \mathrm{MA}<\mathrm{OTH}(\mathrm{p}<0.01), \mathrm{IOD}<\mathrm{IOR}(\mathrm{p}<0.01)$

\&Adjusted for race/ethnicity, smoking status, iodine sufficiency status, age, survey year, body mass index, poverty income ratio, urine creatinine as well as individual food consumption and total nutrient variables as listed in Table 3

\#Adjusted for gender, smoking status, iodine sufficiency status, age, survey year, body mass index, poverty income ratio, urine creatinine as well as individual food consumption and total nutrient variables as listed in Table 3

${ }^{\&}$ Adjusted for gender, race/ethnicity, iodine sufficiency status, age, survey year, body mass index, poverty income ratio, urine creatinine as well as individual food consumption and total nutrient variables as listed in Table 3

\#Adjusted for gender, race/ethnicity, smoking status, age, survey year, body mass index, poverty income ratio, urine creatinine as well as individual food consumption and total nutrient variables as listed in Table 3

between total fiber consumed and ETD $(\beta=0.0075, \mathrm{p}<0.01)$. There was also a positive association between total folates consumed and $\operatorname{ETL}(\beta=0.0002, \mathrm{p}<0.01)$.

Effect of gender, race/ethnicity, smoking and iodine sufficiency statuses

Males had statistically significantly higher levels of DAZ, DMA, EQU, ETD, and ETL $(\mathrm{p}<=0.03$, Table 4$)$. Both NHW and NHB had statistically significantly higher levels of DMA than MA ( $\mathrm{p}<$ 0.01 , Table 4). NHW had higher levels of EQU as well as ETD than both NHB and MA ( $p<0.01$, Table 4). Nonsmokers had statistically significantly higher levels of DMA ( $p=0.01$, Table 4$)$, EQU $(p=0.04$, Table 4), ETD ( $p<0.01$, Table 4$)$, ETL ( $<<0.01$, Table 4$)$ than smokers. Iodine deficiency was associated with statistically significantly lower levels of DAZ ( $\mathrm{p}<0.01$, Table 4), DMA ( $\mathrm{p}=0.01$, Table 4), EQU ( $\mathrm{p}<$ 0.01 , Table 4$)$, and GNS ( $\mathrm{p}<0.01$, Table 4$)$.

\section{Discussion}

New to this study, for the first time, the association of iodine sufficiency status as determined by the observed iodine levels in urine with the observed levels of six phytoestrogens in urine was evaluated. Urinary iodine levels were found to have a statistically significant association with the levels of all six phytoestrogens. Iodine deficiency was associated with statistically significantly lower levels of DAZ, DMA, EQU, and GNS $(\mathrm{p}<0.01)$. For the data used for this study, Spearman correlations between serum cotinine and DAZ, DMA, EQU, ETD, ETL, and GNS were found to be $-0.01,-0.06,-0.04,-0.11$, -0.17 , and 0.003 respectively. These correlations are similar to what have been reported by Ryback et al. [14]. Also, Spearman correlations between urinary iodine and DAZ, DMA, EQU, ETD, ETL, and GNS were found to be $0.23,0.15,0.42,0.18,0.15$, and 0.23 respectively. These correlations are substantially higher than the correlations that serum cotinine had with the phytoestrogens studied here. Thus, it is of importance to consider the role that iodine sufficiency status plays in affecting the observed levels of phytoestrogens. Iodine sufficiency status may affect bioavailability of certain phytoestrogens. A mechanism how this effect may take place is unknown and will need more explanation. This is an area that needs more work, possibly in laboratory animals.

Ryback et al. found unadjusted levels of DMA $(\mathrm{p}<0.01)$ and ETL ( $p<0.01)$ to be statistically significantly higher among nonsmokers as compared to smokers [14]. However, in this study, when adjustments were made for factors that affect levels of phytoestrogens, in addition to DMA and ETL, nonsmokers were also found to have statistically significantly higher levels of EQU and ETD $(\mathrm{p}<=0.04)$ than smokers. Kikkinen et al. found current female smokers to have statistically significantly lower levels of serum ETL than female nonsmokers [17]. It is possible that certain constituents in tobacco smoke may induce enzymes that may accelerate excretion of certain phytoestrogen metabolites or alternatively, smoking may negatively affect bioavailability of certain phytoestrogens. The mechanisms by which tobacco smoke constituents and iodine deficiency affect levels of phytoestrogens are not well understood and will need additional research.

Because of possible changes in diet and metabolism as people age, it is important to consider how age may affect the observed levels of phytoestrogens. Ryback et al. found age to be negatively associated 
Citation: Jain RB. Impact of Dietary and Other Factors Including lodine Sufficiency Status on the Levels of Urinary Phytoestrogens. J Nutri Health. 2016;2(1): 7 .

ISSN: $2469-4185$

with unadjusted levels of EQU ( $\mathrm{p}=0.01)$ [14]. This was not confirmed in this study. On the contrary, I found a positive association between age and ETD, ETL, and GNS ( $p<0.01)$. This may be because I used age as a continuous variable in the regression model, Ryback et al presented results based on unadjusted levels of age as a categorical variable [14].

Because of changes in the volume of distribution with changes in BMI, the observed levels of the chemicals of interest may change. Ryback et al. based on BMI as a categorical variable, found a positive association between BMI and DAZ and between BMI and DMA [14]. These results were not confirmed by this study. Instead, a negative association between BMI with both ETD and ETL was observed $(\mathrm{p}<=0.01)$. Dietary patterns associated with socioeconomic status may affect the observed levels of the chemicals of interest. Higher unadjusted levels of EQU, DMA, ETD, and ETL were associated with increasing PIR [14]. These results were confirmed for ETD and ETL only in this study $(\mathrm{p}<0.01)$.

Both gender and race/ethnicity are known to affect metabolism of many drugs. Ryabck et al. found males to have statistically significantly higher unadjusted levels of DAZ ( $\mathrm{p}=0.02)$ but after adjustments for other factors, for this study, adjusted levels of not only DAZ $(\mathrm{p}<0.01)$ but also DMA ( $p<0.01)$, EQU ( $p=0.02)$ ETD $(p<0.01)$, and ETL ( $p=$ 0.03 ) were found to be higher for males as compared to females [14]. Peeters et al. reported female vegans and vegetarians to have higher levels of ETL than male vegans and vegetarians [18]. Also, Kikkinen et al. reported median serum ETL levels among females to be higher than among males (16.6 nmol/L vs. $13.8 \mathrm{nmol} / \mathrm{L})$ in a Finish study [17]. Ryback et al. found statistically significant differences by race/ ethnicity for unadjusted levels of DAZ, EQU, and DMA [14]. Similar results were found in this study also. Among three major racial/ethnic categories, MA had the lowest adjusted levels of DAZ, DMA, and EQU in this as well as the study by Ryback et al. [14]

Using data from NHANES for 2001-2004, Frankenfeld found positive association between DAZ and consumption of legumes, nuts, and seeds $(p=0.03)$ and grain products $(p<0.01)$ [19]. While Frankenfeld used original 9 USDA food groups, in his study, I converted these 9 food groups into 17 food groups but there were some similarities in the results [19]. In this study, DAZ levels were statistically significantly positively associated with the consumptions of eggs, legumes and nuts, grains, fats and oils, and dried fruits $(\mathrm{p}<=$ 0.02 ). Frankenfeld found positive association between GNS and the consumptions of legumes and nuts seeds, and grain products $(\mathrm{p}<$ $0.01)$ [19]. I also found similar results $(\mathrm{p}<0.01)$. Frankenfeld also found positive association between DMA and the consumption of legumes and nuts, and seeds $(\mathrm{p}=0.02)$ [19]. In this study, in addition to legumes and nuts $(\mathrm{p}<0.01)$, positive association between DMA with the consumptions of eggs ( $\mathrm{p}<0.01$ ), grains $(\mathrm{p}<0.01)$, and fats and oils was also discovered. Both Frankenfeld and I found positive association between EQU and consumptions of milk and milk products $(p<0.01)[19]$. In addition, I found a positive association between EQU and the consumption of seeds $(p=0.03)$ and a negative association between EQU and the consumption of fruits excluding berries and dried fruits $(p=0.01)$. In males, Kikkinen et al. found positive association between serum ETL and the consumptions of the wholegrain products and fruits and berries [17]. In females,
Kikkinen et al. found positive association between serum ETL and the consumptions of vegetables [17]. In this study, only the consumption of eggs was found to have a positive association with urinary ETL. It is possible that associations with dietary variables that phytoestrogens may vary from one matrix to another matrix.

In summary, (i) smoking is associated with $42.9 \%, 18.2 \%, 41 \%$, and $72 \%$ lower levels of DMA, EQU, ETD, and ETL respectively, (ii) iodine deficiency is associated with $47 \%, 55.6 \%, 88.6 \%, 4.5 \%$, $17.8 \%$, and $29.2 \%$ lower levels of DAZ, DMA, EQU, ETD, ETL, and GNS respectively, (iii) consumption of milk products is positively associated with urinary levels of EQU, (iv) consumption of fish is positively associated with urinary levels of ETD, (v) consumption of eggs is positively associated with urinary levels of DAZ, DMA, and ETL, (vi) consumption of legumes and nuts as well as grains is positively associated with urinary levels of DAZ, DMA, and GNS, (vii) consumption of seeds is positively associated with urinary levels of EQU and ETD, (viii) consumption of fruits excluding berries and dried fruits is negatively associated with urinary levels of EQU, (ix) consumption of fats and oils is positively associated with urinary levels of DAZ and DMA, (x) there was a negative association between the total cholesterol consumed and DAZ and DMA, (xi) total fiber consumed was positively associated with ETD, and (xii) total folate consumed was positively associated with ETL.

\section{References}

1. Turner JV, Agatonovic-Kustrin S, Glass BD (2007) Molecular aspects of phytoestrogen selective binding at estrogen receptors. J Pharm Sci 96: 18791885.

2. Dueregger A, Heidgger I, Ofer P, Perktold B, Ramoner R, et al. (2014) The use of dietary supplements to alleviate androgen deprivation therapy side effects during prostate cancer treatment. Nutrients 6: 4491-4519.

3. Schabath MB, Hernandez LM, Wu X, Pillow PC, Spitz MR (2005) Dietary phytoestrogens and lung cancer risk. JAMA 294: 1493-1504.

4. Manach C, Scalbert A, Morand C, Remsey C, Jimenez L (2004) Polyphenols: food sources and bioavailability. Am J Clin Nutr 79: 727-747.

5. Adlecreutz H (2007) Lignans and human health. Crit Rev Clin Lab Sci 44 483-525.

6. Zhang X, Shu XO, Gao YT, Yang G, Li Q, et al. (2003) Soy food consumption is associated with lower risk of coronary heart disease in Chinese women. $J$ Nutr 133: 2874-2878.

7. Villegas R, Gao YT, Yng G, Li HL, Elasy TA, et al. (2008) Legume and soy food intake and the incidence of type 2 diabetes in Shangai Women's Health Study. Am J Clin Nutr 87: 162-167.

8. Dong JY, Qin LQ (2011) Soy isoflavones consumption and risk of breast cancer incidence or recurrence: a meta-analysis of prospective studies. Breast Cancer Res Treat 125: 315-323.

9. Buck K, Zaineddin AK, Vrieling A, Linseisen J, Chang-Claude J (2010) Metaanalysis of lignans and enterolignans in relation to breast cancer risk. Am J Clin Nutr 92: 141-153.

10. Qu XL, Fang Y, Zhang M, Zhang YZ (2014) Phytoestrogen intake and risk of ovarian cancer: a meta-analysis of 10 observational studies. Asian Pac J Cancer Prev 15: 9085-9091.

11. Yan L, Spitznagel EL (2010) Soy consumption and prostate cancer risk in men: a revisit of a meta-analysis. Am J Clin Nutr 89: 1155-1163.

12. Jeng HA, Kantaria K, Beydoun HA (2015) Urinary phytoestrogens in relation to metabolic disturbances among children and adolescents. J Environ Sci Health B 50: 121-127.

13. Acharjee S, Zhou JR, Elajami TK, Welty FK (2015) Effect of soy nuts and 
Citation: Jain RB. Impact of Dietary and Other Factors Including lodine Sufficiency Status on the Levels of Urinary Phytoestrogens. J Nutri Health. 2016;2(1): 7.

equol status on blood pressure, lipids and inflammation in postmenopausal women stratified by metabolic syndrome status. Metabolism 64: 236-243.

14. Ryback ME, Sternberg MR, Pfeiffer CM (2013) Sociodemographic and lifestyle variable are compound- and class-specific correlates of urine phytoestrogen concentrations in the U.S. population. J Nutr 143: 986S-994S.

15. USDA (2008) USDA Food and nutrient database for dietary studies, 3.0 Beltsville, MD. U.S. Department of Agriculture, Agricultural Research Service, Food Surveys Research Group.

16. USDA (2010) USDA Food and nutrient database for dietary studies, 4.1.
Beltsville, MD. U.S. Department of Agriculture, Agricultural Research Service, Food Surveys Research Group.

17. Kikkinen A, Stumpf K, Pietinen P, Valsta LM, Tapanainen H, et al. (2001) Determinants of serum enterolactone concentration. Am J Clin Nutr 73: 10941100.

18. Peeters PH, Slimani N, van der Schouw YT, Grace PB, Navarro C, et al. (2007) Variations in plasma phytoestrogen concentrations in European adults. J Nutr 137: 1294-1300.

19. Frankenfeld CL (2011) Dairy consumption is a significant correlate of urinary equol concentration in a representative sample of US adults. Am J Clin Nutr 93: 1109-1116. 